\title{
The Research on Related Scheduling Model and Algorithm for Logistics Matching Problem
}

\author{
CAO DanTing ${ }^{1,}$, FENG JinZhan ${ }^{2}$ \\ ${ }^{1}$ Jiangxi College of Foreign Studies, Business Administration Department, Jiangxi, Nanchang \\ 330000, China \\ a caodanting@yeah.net
}

Keywords: Scheduling Model; Logistics Matching; Multi Depots Vehicle

\begin{abstract}
The rapid development of business activities to accelerate the development of logistics industry. As the global economy and the advancement of electronic business logistics costs in the proportion of the whole cost is more and more big, the effectiveness of the logistics problems have become more and more prominent. As information technology, communication technology and the development of artificial intelligence technology, fully tap existing logistics resources potential, improve logistics resource utilization, conform to the requirements of the current development. Detail in this paper, the logistics in the matching problem of vehicle scheduling problem is studied, and effective integration of vehicle transportation, goods and transport resources effectively, make full use of limited resources of vehicles, improve the quality of service, cost savings, reduce the energy consumption and reducing exhaust gas pollution, achieve the sustainable development of social and economic benefits.
\end{abstract}

\section{Introduction}

As China's market economy gradually established, transport goods tend to be more multiple varieties and small batch, user, wide distribution, and the corresponding transport vehicle is more for individual businesses, lead to goods transportation company, small, scattered, weak situation [1] The supply of goods scattered, the arrival of the destination, imperfect network, and poor information communication is difficult to satisfy the customer to the present situation of the freight rate and high quality requirements, causing the vehicle's empty loading rate is high, high transport costs, poor transportation company benefit. How to make cars, cargo and integration of the route and reduce the empty, reduce costs, improve operational efficiency, the maximum to meet customer demand, using modern technology for vehicle scheduling method scientifically is the key to solve the above problems. As information technology, communication technology and the development of artificial intelligence technology, fully tap existing logistics resources potential, improve logistics resource utilization, conform to the requirements of the current development.

Modern logistics is put forward that makes it possible to quickly raise the level of logistics in our country. The so-called modern logistics, modern logistics) refers to the information, transportation, warehousing, storage, handling, logistics activities together a kind of management mode, its basic task is to reduce logistics costs, improve the quality of logistics service [2]. The development of information technology and communication technology makes increasing visibility and intelligent logistics process, logistics participants in the functions of organization in the process of logistics coordination and cooperation be strengthened. At the same time, optimization technology and intelligent technology development and introduction of logistics process from the traditional semi-automatic to automation, and intelligent.

\section{The proposed multi depots vehicle scheduling problem}

Customer demand is to realize the movement of the goods in a certain time scope. According to the nature of the transport business, the end can put it into shipping business, shipping business, set to send three integration business. Shipping business refers to the business need to take the goods from the customer designated as the goods sent to another place distribution center; Delivery business 
refers to the business need to deliver the goods from the distribution center to the customer designated another place; Set to send integrated business refers to a business, the beginning and end of the goods are not distribution center business. According to a single distribution center distribution mode - the more distribution center distribution model - more distribution center distribution mode, and the classification of distribution business, on the basis of the evolution of the integrated model, put forward the detail shipping mode, the resulting Multi Depots Vehicle Scheduling Problem [3-4]. Order between the yard and the hubs diagram is shown in figure 1.

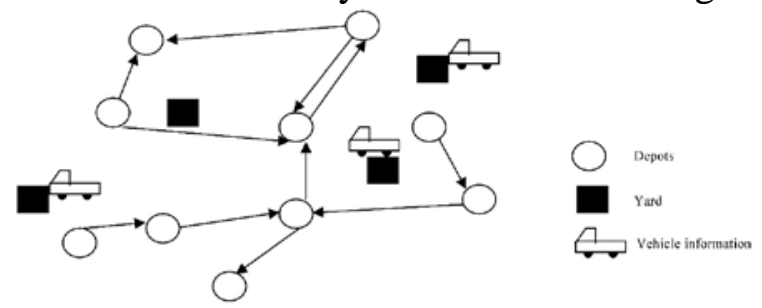

Figure 1. Order diagram of the multi depots

Hubs can be loading and unloading, and can be accessed many times; the arrow in this picture by the end of the starting point to the goods, the direction of the arrows represent the corresponding order. Figure in detail order relation characteristics are as follows: goods and deliver goods orders can be brought into each; some detail between all have the goods transported to each other, the convection of goods; some of the hubs only deliver goods; some of the hubs, only brought into goods.

\section{The establishment of multi depots vehicle scheduling model}

This paper explored the direction to strive to create detailed vehicle scheduling model, through improved ant colony algorithm, ant colony algorithm and hybrid algorithm of particle swarm, detail application and solve the stowage scheduling problems, and improve the efficiency of the vehicle loading rate, transportation resource use and reduce the cost of car transportation. Detail vehicle scheduling process as shown in figure 2.

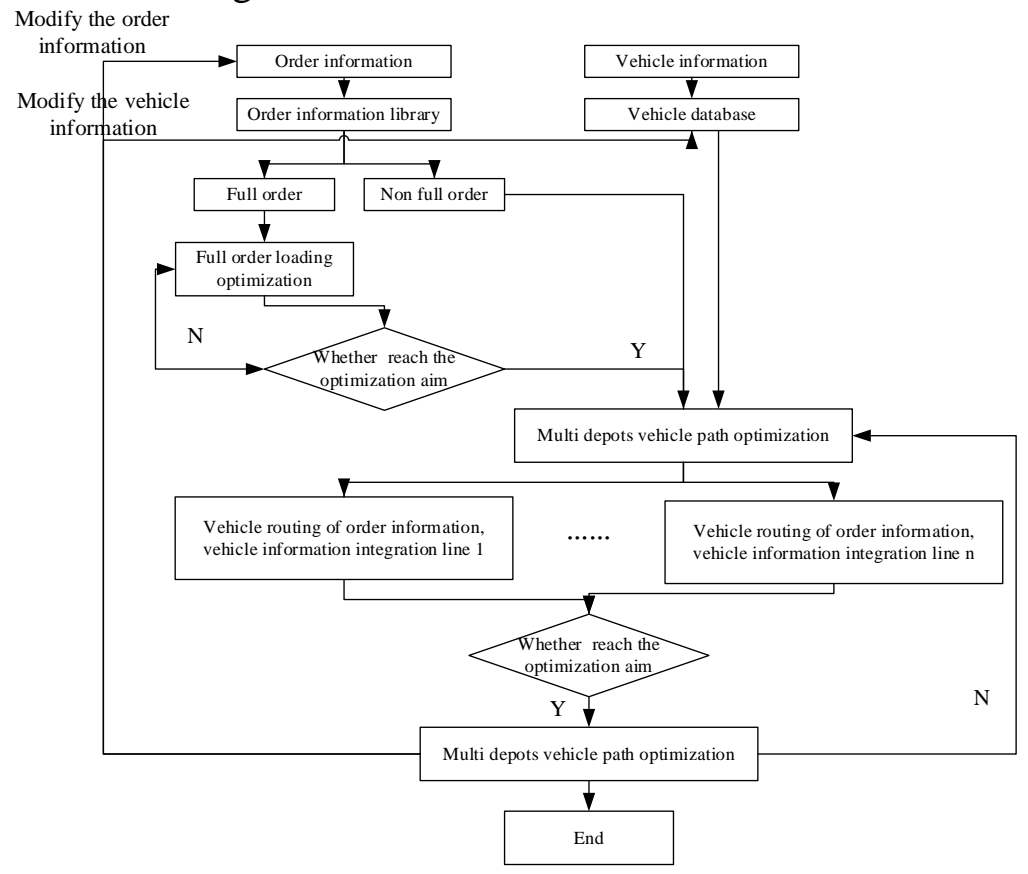

Figure 2. Path optimization process of multi depots vehicle

Consider the objective function and constraint conditions, the multi depots vehicle scheduling model is as follows: 


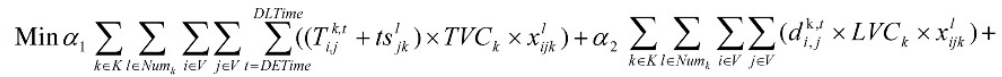

$$
\begin{aligned}
& \alpha_{3} \sum_{k \in K}\left(\mathrm{Num}_{k} \times F C_{k}\right)+\alpha_{4} \sum_{j \in V} \sum_{i \in V} \sum_{i \in \in \mathbb{N}} p_{i j j}^{i a} D T_{i n j}+\alpha_{5} \sum_{j \in V} \sum_{i \in V} \sum_{i \in \in \mathbb{N}} p_{i j j}^{e} W T_{i j j} \\
& \text { Constraints are as follows: } \\
& \sum_{i n \in \mathbb{N}} \sum_{j \in V} \sum_{k \in K} \sum_{l \in N u m_{k}} o n v_{i n j, k}^{l}=i N \\
& \sum_{i \in V} \sum_{k \in K} \sum_{l \in N_{i u m} m_{k}} x_{i j k}^{l}=\sum_{i \in V} \sum_{k \in K} \sum_{l \in N u m_{k}} x_{j i k}^{l} \\
& S S T_{i m j}=\operatorname{Max}\left[A T_{i n j}, e_{i n j}\right], \quad i \in F \\
& \sum_{l \in \text { Num }_{k}} s_{m k}^{l} \leq \text { Num }_{m k} \quad \forall k \\
& \sum_{k \in K} \sum_{l \in N u m_{k}} V_{m k}^{l}=P S V_{m} \leq P S_{m}=P S V_{m}+P S R_{m} \\
& \sum_{i \in v} \sum_{i n \in i N k \in K} \sum_{l \in N_{u m} m_{k}} x_{\text {inmk }}^{l}+P S V_{m} \leq P S_{m} \\
& \sum_{m \in M l \in N u m_{k}} \sum_{m k}^{l}+\sum_{m \in M l \in N u m} \sum_{m k}^{l}=\text { Num }_{k} \\
& \operatorname{Max}\left\{A T_{i m m}\right\} \leq \text { DLTime }_{m} \quad \forall l, \forall k, \forall m \\
& \text { DLTime }_{m} \leq S S T_{m} \\
& A T_{m} \leq \text { DETime }_{m} \\
& \sum_{i \in V} \sum_{j \in V}\left(d_{i j} x_{i j k}^{l}\right) \leq V L_{k, \max }
\end{aligned}
$$

Model parameters and other parameters in the model of this study is the essential difference between the hubs with Order $_{\text {inj }}$, $i$ need to be sent to the $n$ order goods orders, the relationship between the hubs $j$ said, detail the vehicle scheduling model is established, refinement, relations between freight collection of goods, delivery to distinguish the desalt, the vehicle's cargo load fluctuations.

\section{The Adaptive based algorithm in the logistics match}

Genetic algorithm is a simulation of biological heredity and evolution of the natural environment and the probability of the formation of a global optimization method to search. It uses a natural evolution of the model, from the initial population, according to evolution, survival of the fittest natural law, on behalf of the evolutionary adaptation better solution [5]. The basic flow shown in Figure 1.

Adaptive is the basic idea of crossover probability and mutation probability can change as the change of fitness. When the population of each individual fitness in a consistent or local optimum, increase the crossover probability and mutation probability, so as to avoid falling into local optimum, which in turn lead to premature phenomenon; When a relatively scattered groups of each individual fitness, reduce crossover probability and mutation probability, it is not easy to damage excellent individuals, to facilitate good individual preserved. At the same time on the individual selection of fitness is higher than the mean fitness smaller crossover probability and mutation probability, make individual preserved; Those below the population average fitness of the individual, select the crossover probability and mutation probability, on the one hand, poor will be part of the fitness of the individual, on the other hand increase the new individual. In the adaptive genetic algorithm, crossover probability and mutation probability adjust formula 13, 14 by the following formula: 


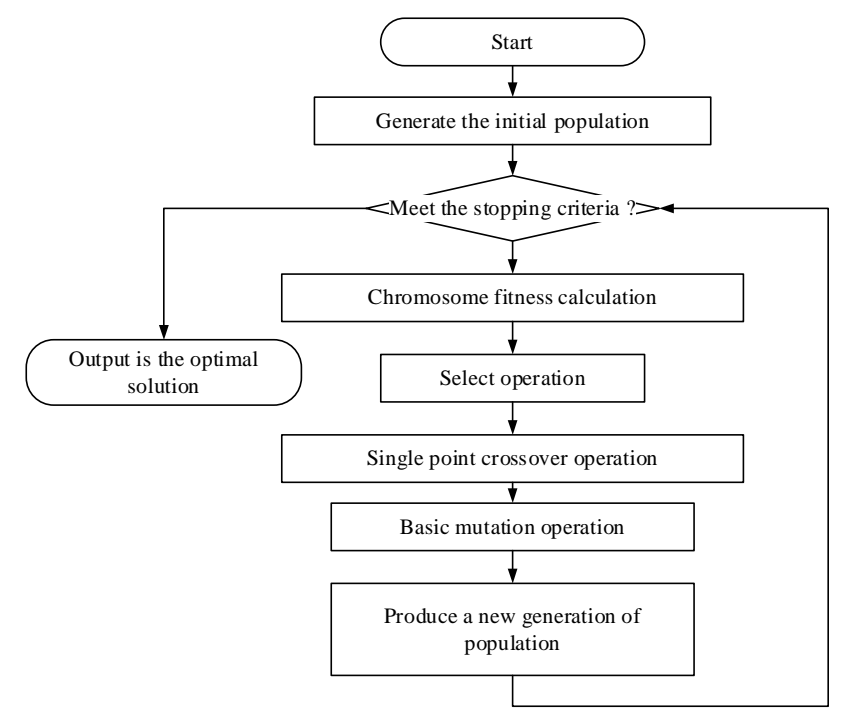

Figure 3. Basic process of genetic algorithm

$$
\begin{aligned}
& \mathrm{P}_{c}= \begin{cases}\frac{k_{1}\left(\mathrm{f}_{\max }-\mathrm{f}^{\prime}\right)}{\mathrm{f}_{\max }-\mathrm{f}_{\operatorname{avg}}}, & \mathrm{f}^{\prime} \geq \mathrm{f}_{\text {avg }} \\
k_{2}, & \mathrm{f}^{\prime}<\mathrm{f}_{\text {avg }}\end{cases} \\
& \mathrm{P}_{\mathrm{m}}= \begin{cases}\frac{k_{3}\left(\mathrm{f}_{\max }-f\right)}{\mathrm{f}_{\max }-\mathrm{f}_{\operatorname{avg}},}, & \mathrm{f}^{\prime} \geq \mathrm{f}_{\text {avg }} \\
k_{4}, & \mathrm{f}^{\prime}<\mathrm{f}_{\text {avg }}\end{cases}
\end{aligned}
$$

( $\mathrm{f}_{\max }$ is the biggest fitness for the population; $\mathrm{f}_{\text {avg }}$ is the population average fitness; $\mathrm{f}^{\prime}$ is to participate in cross of the two The fitness of individuals in larger individuals; $f$ is the fitness of individual variation)

\section{Conclusion}

Vehicle scheduling problem is a key part of modern logistics service supply chain optimization, supply chain logistics services also spend the most part of the cost of service, while the vehicle scheduling problem is a modern e-commerce activities conducted indispensable support section. Vehicle scheduling problem and solving algorithms Logistics matching system is the development of intelligent transport systems, building integrated logistics systems and carry out basic e-commerce. Therefore, further study of the logistics matching vehicle scheduling problem, a higher scientific and engineering application value.

\section{Acknowledgements}

Humanity and Social Science Youth foundation of Education Department of Jiangxi, JJ1348."Optimization of the internal structure of the logistics industry under the energy crisis environment from the analysis of deconstruction."

\section{References}

[1] C.C. Tsai: Expert Systems with Applications, Vol.36 (2009) No.5, p. 9506.

[2] L. Wei-hua, X. Xue-cai, R. Zheng-xu: Supply Chain Management: An International Journal, Vol.16 (2011) No.6, p. 391.

[3] E. Levner, V. Kats: Computers \& Industrial Engineering, Vol.59 (2010) No.2, p. 352.

[4] D. Ivanov: International Journal of Production Research, Vol.47 (2009) No.5, p.1153.

[5] V. Pillac, M. Gendreau, C. Guéret: European Journal of Operational Research, Vol.225 (2013) No.1, p. 1. 